\title{
Studies on the dielectric properties of olive pomace grains reinforced polymer matrix composites
}

\author{
L. KREIT ${ }^{* 1}$, Z. SAMIR ${ }^{1}$, M.E. ACHOUR ${ }^{1}$, A. OUERIAGLI' ${ }^{2}$, A. OUTZOURHIT ${ }^{2}$, \\ L.C. $\operatorname{COSTA}^{3}$, M. MABROUKI ${ }^{4}$ \\ ${ }^{1}$ LASTID Laboratory, Sciences Faculty, Ibn Tofail University, Kenitra, Morocco \\ ${ }^{2}$ LPSCM Laboratory, FSS, Cadi Ayyad University, Marrakech, Morocco \\ ${ }^{3}$ I3N and Physics Department, University of Aveiro, Aveiro, Portugal \\ ${ }^{4}$ Laboratory LGI, FST, Sultan Moulay Slimane University, Beni Mellal, Morocco \\ *Corresponding author: E-mail: kreit.lamyaa@gmail.com
}

Keywords: Biocomposites, Dielectric Relaxation, Kramers-Kronig Relation

\begin{abstract}
In this work, the polyester polymer matrix filled with olive-pomace grains was investigated by means of dielectric spectroscopy in the frequency range $10 \mathrm{KHz}-1 \mathrm{MHz}$ and temperature from $300 \mathrm{~K}$ to $400 \mathrm{~K}$. A numerical Kramers-Kronig transformation allowed the calculation of dielectric losses from the measured dielectric constant. The obtained relaxation processes were attributed to the orientation polarization imputed to the presence of polar water molecules in the pomace grains and to the accumulation of charges at the pomace grains/polyester interfaces. The analysis of the temperature dependence of the relaxation time using the Arrhenius equation shows the existence of two activation energies which are attributed to contributions of different relaxation processes.
\end{abstract}

\section{Introduction}

Over the last few years, natural fibers have become the main reinforcement for high performance composite materials [1]. The uses of lignocellulosic fibers have ranged from the construction industry to the automotive industry. The main attraction of bio-fiber reinforced composites lie in their low density and high strength. The most interesting aspect about natural fibers is their positive environmental impacts. As they come from a natural resource, they are a completely biodegradable and nonabrasive material and can be easily eliminated after the degradation of the polymer. The high sound attenuation of lignocellulosic composites is another added advantage. Vegetable fibers also provide a relativity reactive surface, which can be used for grafting specific groups and inducing new functional entities. In addition, the recycling by combustion of polysaccharide filled composites is easier in comparison with inorganic fillers [2].

Relaxation phenomena is one of the most studied topics in materials sciences and the spectra of the real and imaginary complex permittivity are used extensively to present this kind of dielectric phenomenon. In our work, the analysis of relaxation phenomenon may be rendered difficult because of dc-conductivity contribution that hides any dielectric relaxation process [3]. In order to overcome this shortcoming caused by high electrical conductivity, an alternative method, consisting of using the representation of the ohmic conduction free based on the derivation has been introduced in this paper. Furthermore, using the Arrhenius equation, the analysis of the temperature dependence of the relaxation time shows the existence of two activation energies which are attributed to contributions of relaxation processes. The mechanisms that induce relaxation processes are not yet clearly understood. So, it is appropriate to investigate how the dielectric properties of such bio-fiber reinforced composites are affected by the additional polarization effects caused by the presence of nanoparticles. 


\section{Experimental details}

\section{Materials}

We used the natural olive-pomace grains of $63 \mu \mathrm{m}$ size, loaded in a polyester polymer matrix, forming pellets with $13 \mathrm{~mm}$ diameter, and thickness of about $3 \mathrm{~mm}$. The matrix used in this work was unsaturated polyester resin $154 \mathrm{~TB}$, including $31 \mathrm{wt} \%$ of styrene monomer, requiring 30 minutes for gelation, at constant $\mathrm{T}=300 \mathrm{~K}$, and was acquired from Cray Valley/Total, USA. The pomace/polyester nanocomposite has been prepared by mixing $5.87 \mathrm{~g}$ of liquid polyester resin and $0.2 \%$ weight of cobalt octanone, as reaction activator. Pomace particles were introduced in different fractions, before adding $1 \%$ of hardener to make each mixture cohesive. The methyl ethyl ketone peroxide (MEKP) is used to harden the mixtures and to facilitate fabrication process. Each olive pomace/Polyester composite was mixed at room temperature for 5 minutes, promoting the gelation and after it was poured into the mold. After two hours the samples were unmolded, taking 24 hours for having a complete polymerization.

\section{Dielectric measurements}

The complex permittivity function, $\varepsilon^{*}=\varepsilon^{\prime}-i \varepsilon^{\prime \prime}$, was measured by using a Hewlett Packard Network Analyzer (Model 4192A, USA). The dielectric constant $\varepsilon^{\prime}$ and the loss factor $\varepsilon^{\prime \prime}$ of the samples were calculated from the admittance $Y^{*}=G+i B=i C_{0} \omega \varepsilon^{*}$ of the equivalent circuit leading to $\varepsilon^{\prime}$ $=2 h B / \varepsilon_{0} d^{2} \pi^{2} F$, where $B$ is the susceptance, $G$ the conductance, $F$ the frequency, $\varepsilon_{\mathrm{o}}$ the vacuum dielectric constant, and $h$ and $d$ are the thickness and the diameter of the sample, respectively. The measurements were performed in the frequency range $10 \mathrm{KHz}-1 \mathrm{MHz}$ under isothermal conditions for temperatures ranging between 300 and $400 \mathrm{~K}$.

\section{Results and discussion}

The Figure 1 shows the real and the imaginary parts of the complex permittivity of the olive pomace/Polyester composite at a temperature of $300 \mathrm{~K}$, as a function of the frequency and for different concentrations of the olive-pomace. The both real and imaginary parts increase with the olive pomace and display a dispersive behavior versus frequency. The analysis of relaxation processes is often hampered due to the ohmic conduction losses that obscure any relaxation process [4]. This problem was observed in this composite. However, it is still possible to work around this issue by using the Kramers-Kronig relation [5]. This technique eliminates the ohmic conduction from the measured loss spectra by using an elegant way based on inversion technique, i.e. on calculating the imaginary part from the real part [Eq. (1)][6-8]:

$$
\varepsilon_{\text {deriv }}^{\prime \prime}=-\frac{\pi}{2} \frac{\partial \varepsilon^{\prime}(\omega)}{2 \partial \ln \omega} \approx \varepsilon^{\prime \prime}
$$

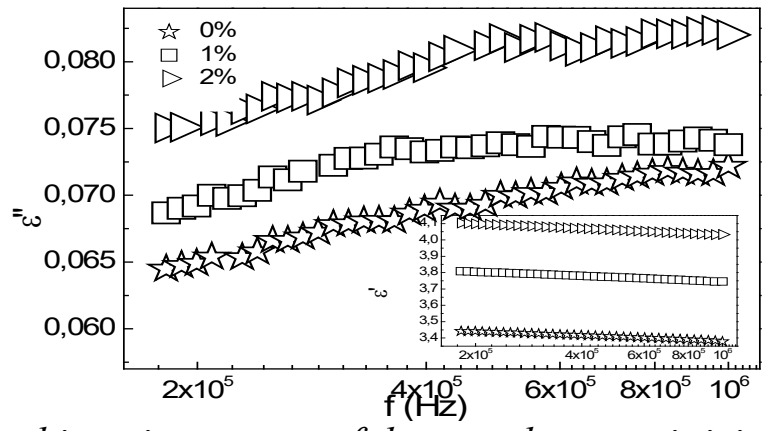

Fig. 1. real part (inset) and imaginary part, of the complex permittivity for different concentrations of the olive-pomace, at a temperature of $300 \mathrm{~K}$.

As one can see in Figure 2 and Figure 3, this inversion technique allowed us to obtain conductivity free dielectric loss peaks, which are a clear signature of the relaxation in the composites. This 
relaxation is associated to polarization of the water molecules linked to cellulosic olive pomace grains which formed a monomolecular layer wrapping the external surface of the grains. Water molecules are known to be tightly bound the surface hydroxyl group of cellulose olive pomace grains. The analysis of the obtained results shows the influence of the temperature and concentration of olive-pomace grains on the dielectric properties of the composite [9].

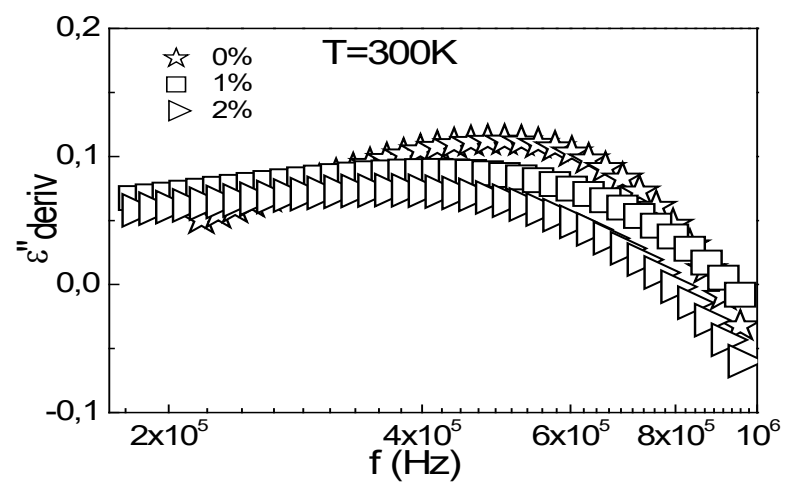

Fig. 2 .Variation with frequency of the ohmic conduction free dielectric loss, $\varepsilon_{\text {deriv }}^{\prime \prime}$, for concentrations of the olive-pomace $0.0,1.0$ and $2.0 \%$, at a temperature of $300 \mathrm{~K}$.

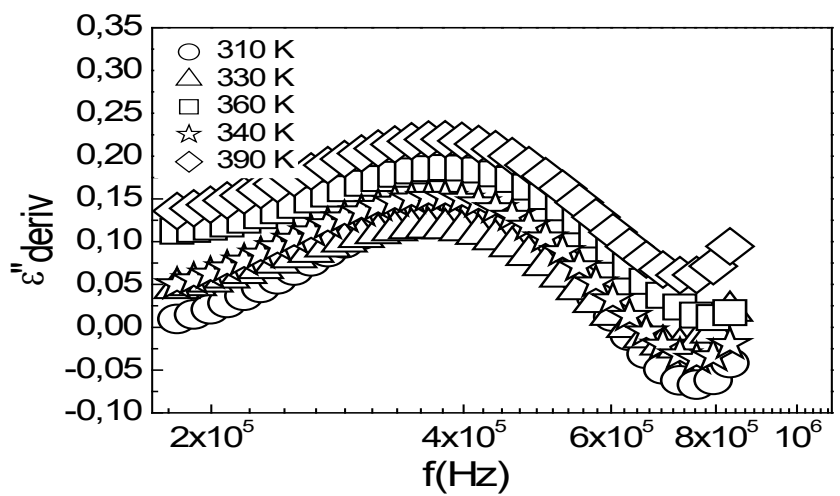

Fig. 3. Ohmic conduction free, $\varepsilon^{\prime \prime} d e r i v$, at various temperatures for a concentration of the olivepomace $(\phi=2.0 \%)$.

In order to further elucidate the dielectric relaxation in the olive pomace/Polyester, it is important to estimate the activation energy associated with the relaxation process which could be obtained from the temperature dependence frequency, according to the relation [Eq. (2)]:

$$
f_{\max }=f_{0} e^{-\frac{E_{a}}{K_{B} T}}
$$

with $E_{a}, K_{B}$ and $T$ being the activation energy, the Boltzmann's constant and the absolute temperature respectively. It should be noted that the activation energy was calculated from the slopes of the straight lines of $\ln \left(\mathrm{f}_{\max }\right)$ versus $(1000 / \mathrm{T})$ for the concentrations of olive-pomace grains of $0.0,1.0 \%, 2.0 \%$ and $3.0 \%$ represented in Figure 4. 


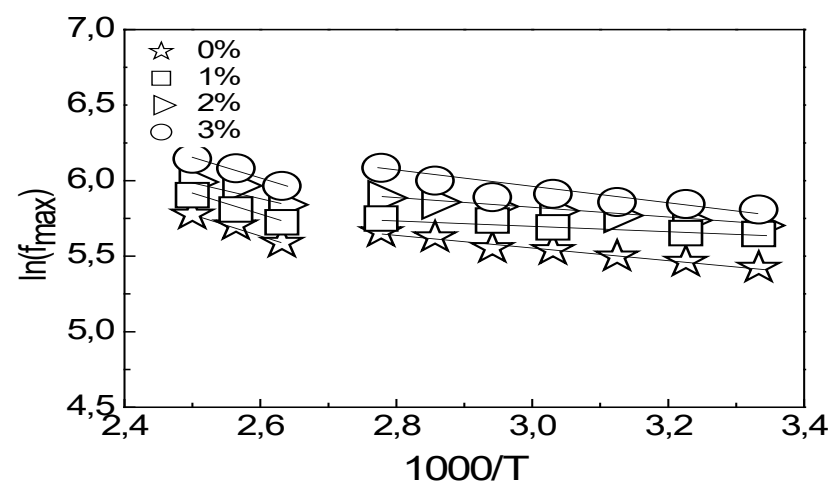

Fig.4. Ln $\left(f_{\max }\right)$ vs. 1000/T for different concentrations of olive-pomace. The solid lines are the least square linear fits to Eq. (3)

The values of activation energies are given in Table 1.

Table1. Activation energies for pomace grains/polyesters composites having 0.0 and 3.0 vol. percentages of pomace grains.

\begin{tabular}{|c|c|c|}
\hline \multirow{2}{*}{$\begin{array}{l}\text { Fraction } \\
\text { Volume }\end{array}$} & \multicolumn{2}{|c|}{ Temperature range } \\
\cline { 2 - 3 } & $300 \mathrm{~K}-370 \mathrm{~K}$ & $380 \mathrm{~K}-400 \mathrm{~K}$ \\
\hline $0.0 \%$ & $0.0019 \mathrm{eV}$ & $0.0053 \mathrm{eV}$ \\
\hline $1.0 \%$ & $0.0014 \mathrm{eV}$ & $0.0042 \mathrm{eV}$ \\
\hline $2.0 \%$ & $0.0009 \mathrm{eV}$ & $0.0050 \mathrm{eV}$ \\
\hline $3.0 \%$ & $0.0019 \mathrm{eV}$ & $0.0048 \mathrm{eV}$ \\
\hline
\end{tabular}

As seen in Table 1, there are two temperature regions of the maximal frequency in this composite: $300-370 \mathrm{~K}$ and $380-400 \mathrm{~K}$. This behavior can be taken as indication that the olive pomace grains have different energies and structural configurations. As shown in figure 4 there is one straight line for the maximal frequency versus temperature plot at the given concentration are applicable in each temperature region. It is very clear from this figure that there is a strong dispersion of maximal frequency with concentration.

\section{Conclusion}

In the present work investigation regarding the dielectric behavior of polyester matrix and composites based on pomace grains, have been studied over the temperature range from 300 to $400 \mathrm{~K}$ and over the frequency range from $10 \mathrm{KHz}$ to $1 \mathrm{MHz}$. The results were analyzed using the KramersKronig transformation which allows the calculation of dielectric losses from the dielectric permittivity measured. In addition to the relaxation associated to the glass transition of the polyester resin matrix and ionic relaxation caused by the mobility of dielectric charges, the presence of pomace grains in the composite gives rise to other relaxation associated to water polarization. The analysis of the temperature dependence of the relaxation time using the Arrhenius equation shows the existence of two activation energies which are attributed to contributions of different relaxation processes.

\section{Acknowledgment}

The authors acknowledge FCT-CNRST bilateral cooperation, and FEDER by funds through the COMPETE 2020 Program and National Funds through FCT - Portuguese Foundation for Science and Technology under the project UID/CTM/50025/2013 


\section{References}

[1] A. Hodzic, R. Shanks, Natural Fibre Composites: Materials, Processes and Properties; Woodhead Publishing Series in Composites Science and Engineering, Elsevier Science, 2014.

[2] I. Ben Amor, H. Rekik, H.Kaddami, M.Raihane, M.Arous and A.Kallel, "Studies of dielectric relaxation in natural fiber-polymer composites", Journal of Electrostatics 67, 2009, 717722. http://dx.doi.org/10.1016/j.elstat.2009.06.004

[3] Z. Samir, Y. el Merabet, M.P.F. Graça, S. Soreto Teixeira, M. E. Achour, L. C. Costa, "Dielectric behavior of carbon nanotube particles filled polyester polymer composites", J. Comp. Materials, 2016 ( in press). http://dx.doi.org/10.1177/0021998316665682

[4] M. Wuubbenhorst, J. v. Turnhout, "Analysis of complex dielectric spectra. I. One-dimensional derivative techniques and threedimensional modeling", Journal of Non Crystalline Solids, 2002,305, 40-49. http://dx.doi.org/10.1016/S0022-3093(02)01086-4

[5] JR. Macdonald, WR. Kenan, Impedance spectroscopy- Emphasizing solid materials and systems. John Wiley and Sons, New York, Interscience 1987; 368.

[6] P. Steeman, J. Vanturnhout, "Fine structure in the parameters of dielectric and viscoelastic relaxations". Macromolecules 1994, 27(19),5421-5427. http://dx.doi.org/10.1021/ma00097a023

[7] A. Molak, M. Paluch, S. Pawlus, J. Klimontko, Z. Ujma, I. Gruszka, "Electric modulus approach to the analysis of electric relaxation in highly conducting $(\mathrm{Na} 0.75 \mathrm{Bi} 0.25)(\mathrm{Mn} 0.25 \mathrm{Nb} 0.75) \mathrm{O} 3$ ceramics", Journal of Physics 2005, 38(9), 1450-1460. http://dx.doi.org/10.1088/0022-3727/38/9/019

[8] H. Mingjuan, Z. Kongshuang, "Effect of volume fraction and temperature on dielectric relaxation spectroscopy of suspensions of PS/PANI composite microspheres", The Journal of Physical Chemistry C 2008, 112, 19412-19422. http://dx.doi.org/10.1021/jp803530m

[9] Tagmouti, S.E.Bouzit, L.C.Costa, M.P .F .Garça and A.Outzourit "Impedance Spectroscopy of Nanofluids based on Multiwall Carbon Nanotubes", Spectroscopy Letters, 48:10, 761-7666, 2015. http://dx.doi.org/10.1080/00387010.2015.1034874 